\title{
Pluralité des instruments et des logiques politiques pour l'adaptation aux changements globaux en zone côtière
}

\author{
Sandrine Dhénain ${ }^{1, *}$,a et Olivier Barreteau ${ }^{2}$ \\ 1 Sciences de l'environnement, science politique, Irstea, UMR183 G-EAU, Montpellier, France \\ 2 Modélisation, sciences de l'eau, Irstea, UMR183 G-EAU, Montpellier, France
}

Reçu le 23 novembre 2016. Accepté le 8 octobre 2018

Depuis plus d'une dizaine d'années, NSS a publié nombre de textes sur l'adaptation aux changements climatiques. Après avoir rappelé l'origine de ce concept d'adaptation et son appropriation par différentes disciplines scientifiques, Guillaume Simonet $(N S S, 2009,4)$ a montré comment nous sommes passés de l'adaptation au climat à l'adaptation au changement climatique. Michel Damian (NSS, 2007, 4) a analysé comment cette notion est venue à l'ordre du jour de la politique climatique; G. Simonet (NSS, 2015, Supp.) a étudié plus précisément l'évolution conceptuelle au fil des rapports du GIEC. Un dossier (NSS, 2010,3 ) consacré à cette notion a permis, entre autres, d'explorer les enjeux politiques qui y sont attachés et sa prise en charge par différents types d'expertise. Le présent article s'inscrit dans cette même perspective en s'intéressant aux projets politiques de l'adaptation aux changements globaux dans la gestion des territoires littoraux. En s'appuyant sur une grille analytique originale et un travail empirique mené en France, les auteurs identifient divers modèles politiques proposés par des acteurs locaux qui doivent répondre à l'injonction de l'adaptation aux changements climatiques.

La Rédaction

\begin{abstract}
Résumé - L'adaptation au changement climatique est un nouvel enjeu pour la gestion des territoires. Au niveau local, elle apparaît souvent comme une injonction, alors même que, pour l'instant, elle est un concept flou. Elle est présentée comme l'application de bonnes pratiques, mais les questions « qui s'adapte à quoi ?» et «pourquoi?» demeurent implicites. En explicitant ces éléments, nous proposons de montrer que l'adaptation est une question plurielle et politique. À partir de l'analyse des documents de planification et des plans d'action faisant référence aux changements globaux sur un territoire littoral, nous montrons l'existence de quatre logiques d'adaptation distinctes, plus ou moins transformatrices du système socioécologique, que l'on peut appréhender à partir de la typologie suivante : «contrôler et maintenir», «faire faire», «réguler» et «reconfigurer», qui portent en germe différentes reconfigurations socioéconomiques et politiques.
\end{abstract}

Mots-clés : adaptation / changement climatique / gouvernance / action publique locale / littoral

\begin{abstract}
Policy instruments and political dynamics of adaptations to global change in coastal areas. Since the 2000 s, "adaptation" is a new dictate for the management of local territories in France, but its implementation is fairly limited. Adaptation is mainly a semantically unclear and loosely defined concept. Decision-makers could "operationalize" adaptation by simply applying a specific methodology. However, adaptation is not a mere mechanism; it is also a process that implies economic, social and ecological trade-offs for the socio-ecological system. These political dimensions are often unformulated. In order to provide a vehicle to clarify this concept and its political dimensions, we propose a typology of adaptation measures. What does adaptation mean? Adjustment of what (territories, populations, communities, local economies, etc.), to what (climate change, global change) and with what effects? We
\end{abstract}

\footnotetext{
* Auteur correspondant : sandrine.dhenain@smavd.org

${ }^{a}$ Sandrine Dhénain travaille depuis novembre 2017 pour le Syndicat mixte d'aménagement de la vallée de la Durance (SMAVD).
} 
reviewed local actions and strategic plans related to climate but also to urban planning, flooding and water management on the eastern coastal area of Languedoc Roussillon in Mediterranean France. We conducted and analyzed semi-structured interviews with institutional actors. We analyzed and classified public policy instruments, associated the underlying "logic" (raise limiting factors, create a new awareness, etc.), and their potential effects. Throughout our effort to develop a typology, we have highlighted the political dimensions of adaptation actions and shed a light on trade-offs linked to adaptation choices.

Keywords: adaptation / climate change / governance / decision making / coastal areas

L'adaptation est un concept diffus et confus. Très marqué à l'origine par les sciences de la nature qui l'envisagent comme l'évolution des caractéristiques des espèces pour faire face à un environnement changeant, il est désormais associé au changement climatique et est passé au premier plan des concepts mobilisés pour analyser les voies possibles des sociétés pour faire face à celui-ci. Son usage se fait dans de multiples arènes, à la frontière du scientifique et du politique, à différentes échelles et au regard de multiples enjeux, ce qui brouille davantage encore les contours de cette notion ${ }^{1}$. L'injonction selon laquelle «il faut s'adapter» est présentée comme une évidence, relayée par les institutions publiques à l'échelle internationale (Nations unies), européenne et nationale (ministère en charge de l'Écologie et du Développement Durable, agences de l'eau), en s'appuyant sur les recommandations du Groupe d'experts intergouvernemental sur l'évolution du climat (GIEC). Si le changement climatique est une réalité, la manière de s'adapter et la responsabilité de l'action restent floues. La loi du 12 juillet 2010 portant engagement national pour l'environnement ${ }^{2}$, dite Grenelle 2, a ainsi obligé les collectivités locales françaises de plus de 50000 habitants à mettre en place un plan climat-énergie avec un volet adaptation. La loi de transition énergétique pour une croissance verte $^{3}$ (2015) mentionne également l'adaptation et renvoie l'obligation d'élaborer un plan climat-air-énergie aux établissements publics de coopération intercommunale (EPCI) de plus de 20000 habitants. Les récents décrets d'application ne donnent que peu d'éléments sur les modalités du passage à l'action. Sur le terrain, régions, villes, services de l'État, acteurs locaux discutent et

\footnotetext{
${ }^{1} \mathrm{Ce}$ travail est issu d'une thèse CIFRE (Conventions industrielles de formation par la recherche) réalisée à l'Irstea Montpellier (UMR G-EAU) au sein du cabinet TEC Conseil (Marseille). Il a été financé par l'Agence nationale de la recherche (ANR) et le Belmont Forum dans le cadre du programme Multi-Scale Adaptations to Global Change in Coastlines (MAGIC).

${ }^{2}$ Loi $\mathrm{n}^{\circ} 2010-788$ du 12 juillet 2010 portant engagement national pour l'environnement, Journal Officiel de la République française, 0160, 13 juillet 2010, p. 12965.

${ }^{3}$ Loi $\mathrm{n}^{\circ}$ 2015-992 du 17 août 2015 relative à la transition énergétique pour la croissance verte, Journal Officiel de la République française, 0189, 18 août 2015, p. 14263.
}

expérimentent diverses actions : économies d'eau, projets de végétalisation ou encore opérations de recul stratégique en zone littorale. Ces modalités opérationnelles sont censées résoudre l'équation locale de l'exposition et de la sensibilité $^{4}$ pour limiter les conséquences climatiques. Cependant, au niveau local, il est difficile d'isoler l'aléa climatique d'autres changements ${ }^{5}$, notamment démographiques. Ce sont les conséquences possibles de l'ensemble de ces changements qui sont prises en compte dans ces plans d'action et documents de planification. De surcroît, les questions de «qui s'adapte à quoi » et «pourquoi » restent implicites, et les sens et les effets sociaux, économiques et politiques des adaptations sont souvent laissés dans l'ombre. L'objet de cet article est d'expliciter le fait que ces options ne sont pas neutres et qu'elles sont porteuses de représentations spécifiques des enjeux à traiter, de conceptions de la régulation politique et d'arbitrages entre secteurs, enjeux et territoires.

Pour mener cette analyse, nous combinons des travaux sur la résilience, l'adaptation au changement climatique, les systèmes écologiques et sociaux (SES) (Berkes et Folke, 1998) et le concept d'instruments d'action publique (IAP) (Lascoumes et Le Galès, 2005). Nous avons construit une typologie à partir d'un travail empirique mené, d'une part, sur un terrain principal situé sur la côte languedocienne, qui couvre des territoires de schémas de cohérence territoriaux (SCOT) et des bassins versants, et, d'autre part, cinq terrains secondaires qui concernent des villes ou des EPCI situés en zones urbaines, rurales ou littorales dans d'autres régions françaises. Nous y avons analysé les actions locales d'adaptation aux changements globaux, dans la gestion de l'eau, des risques et de l'aménagement du territoire.

La première partie de l'article revisite les multiples définitions du concept d'adaptation à l'aune de la littérature sur la résilience et la vulnérabilité. Elle

\footnotetext{
${ }^{4}$ Dans les documents techniques de l'Ademe, la sensibilité est définie comme la propension d'un territoire à être affecté par l'aléa climatique, en fonction des activités du territoire, du profil démographique, etc.

${ }^{5}$ Le Languedoc-Roussillon est, de 2007 à 2012, la deuxième région métropolitaine au plus fort taux de croissance démographique.
} 
présente aussi les divers éclairages du concept d'IAP appliqué à l'adaptation. La deuxième partie expose la typologie et les axes qui la structurent. Enfin, la troisième partie met en avant leurs apports et leurs limites.

\section{L'adaptation dans le triptyque vulnérabilité - résilience - adaptation}

\section{L'adaptation, capacité des systèmes face aux perturbations}

Résilience, vulnérabilité et adaptation décrivent des états et des caractéristiques des systèmes écologiques et sociaux (SES) et leurs processus d'évolution face à des changements potentiellement rapides, non linéaires et incertains (Smit et al., 1999; Smit et Wandel, 2006; Brooks, 2003).

La résilience est «la capacité à absorber des perturbations et à se réorganiser tout en opérant des changements de manière à conserver essentiellement les mêmes fonctions, structures, identités et rétroactions 》 (Walker et al., 2004). L'analyse de la résilience part de celle des propriétés de fonctionnement du système. L'analyse de la vulnérabilité s'intéresse aux capacités différenciées des sociétés à supporter les conséquences du changement.

La vulnérabilité est définie comme le degré auquel un système, ou un sous-système, est susceptible d'être affecté négativement à la suite d'une exposition à une perturbation ou à un stress en particulier (Turner et al., 2003). Dans les deux cas, l'adaptation est envisagée sous l'angle de « capacités adaptatives ». Du point de vue de la résilience, elles sont portées essentiellement par des individus et des communautés (Walker et al., 2004) et incluent des mécanismes d'auto-organisation (Berkes et Folke, 1998; Folke et al., 2005; Smit et al., 2001), d'innovation et d'apprentissage (Carpenter et al., 2001). Elles permettent de conduire la résilience et de « naviguer » au cours de ces transitions entre les différents «états du système» (Gunderson, 2000; Berkes et al., 2008; Gunderson et al., 2002; Folke, 2006).

L'adaptation est la capacité permettant de réduire la vulnérabilité face aux perturbations (Pielke, 1998). Elle est intentionnelle. L'influence politique, les relations sociales, l'accès aux ressources, l'environnement institutionnel constituent des déterminants de la capacité d'adaptation (Kelly et Adger, 2000 ; Wisner et al., 2004). Ces capacités varient dans l'espace (selon les groupes) et le temps (Smit et Wandel, 2006). L'adaptation est appréhendée comme une capacité de réponse aux perturbations dont on peut dresser la liste des déterminants.

Dans le corpus relatif au changement climatique, l'adaptation, envisagée d'abord comme un état, évolue vers l'idée d'un processus (Smit et al., 1999; Smit et
Wandel, 2006). L'idée de processus invite à porter attention à la fois à la continuité des actions dans le temps, mais aussi au rôle des acteurs dans le passage à l'action, vers la transformation. Cette vision de l'adaptation va s'imposer au fil des rapports du GIEC (Simonet, 2016).

\section{Des nuances dans le degré de reconfiguration du système et les formes de réponses}

De manière générique, l'adaptation est définie comme la «capacité de réponse » (Gallopín, 2006) face à des événements effectifs ou anticipés. Différents qualificatifs sont associés à l'adaptation, ces nuances (Bassett et Fogelman, 2013) traduisant une certaine gradation de l'ampleur de la réorganisation du système qu'implique l'adaptation (Adger, 2006; Turner et al., 2003). Ainsi, l'adaptation est parfois entendue comme synonyme d' «ajustement»: le terme qualifie alors une réponse sur le court terme qui opère des modifications à la marge (Kasperson et Archer, 2005). Plus souvent, l'adaptation, en tant que telle, modifie plus amplement le système à plus long terme (Smit et Wandel, 2006).

La littérature sur la résilience distingue les notions d'adaptation-accommodation et d'adaptation-transformation. L'accommodation renvoie à la fois à l'idée de faire face à des chocs et de les amortir (robustesse et fonction tampon). La transformation, quant à elle, est définie comme une reconfiguration plus structurelle et fondamentale du système (des structures biophysiques, économiques, sociales) (Folke, 2006). Celle-ci peut être involontaire lors du dépassement de seuil, mais aussi volontaire pour échapper à des situations non souhaitables (Walker et al., 2004; Folke et al., 2005; Folke, 2006). L'adaptation recouvre ainsi différentes nuances: faire face aux perturbations, ajuster à la marge et transformer le système. Elle traduit donc des ambitions de changements sociaux, politiques et économiques différenciées.

L'adaptation se caractérise également par ses formes, natures et fonctions répertoriées par diverses typologies (Smit et al., 2000; Cutter et al., 2000; Burton et al., 2002; Burton et al., 1993; Bijlsma et al., 1995; Biesbroek et al., 2010; Smit et al., 2001; Carpenter et al., 2001). Dans ces typologies, qui sont essentiellement théoriques ${ }^{6}$, la dimension politique apparaît souvent en filigrane. Or, l'élaboration de ce qui est «à reconfigurer», "souhaitable», «non souhaitable» est bien une question éminemment politique. C'est pour investiguer cette dimension que des outils théoriques complémentaires sont utiles.

\footnotetext{
${ }^{6}$ Certaines, plus récentes, sont plus empiriques : Biagini et al., 2014.
} 


\section{Regard sur la dimension politique de l'adaptation : l'entrée par les IAP}

La littérature sur l'adaptation aborde les dimensions politiques d'une manière fonctionnaliste. Les droits civiques et politiques sont des déterminants des capacités d'adaptation (Brooks et al., 2005). La gouvernance, les décalages et les conflits potentiels entre échelles de prise de décision peuvent être des barrières à l'adaptation (Brooks et al., 2005; Keskitalo et Kulyasova, 2009). Différents dispositifs institutionnels peuvent être plus ou moins propices à l'adaptation (Smit et al., 2001; Huq et al., 2004). Ces travaux ne considèrent pas le politique comme le fruit de rapports sociaux et minimisent les rapports de pouvoir. Pourtant, les modalités de mise à l'agenda de l'enjeu climatique façonnent les modalités de son traitement aux échelles internationale (Demeritt, 2001; Aykut et Dahan, 2014), nationale et locale (Marquet et Salles, 2014). L'adaptation se constitue comme un nouveau référentiel de gestion (Marquet et Salles, 2014; Richard, 2013), elle devient politique et renouvelle les argumentaires de la négociation dans la gestion de l'eau, par exemple.

La référence aux "instruments d'action publique » (IAP) permet d'aborder ces dimensions. Un instrument peut être défini comme «un dispositif à la fois technique et social qui organise des rapports sociaux spécifiques entre la puissance publique et ses destinataires en fonction des représentations et des significations dont il est porteur » (Lascoumes et Le Galès, 2005; Halpern et al., 2014). Un instrument peut être un outil statistique, une carte scolaire ou une carte des risques, une taxe carbone, etc. Ce ne sont pas des outils neutres et indifféremment disponibles, ils sont porteurs d'un rapport au monde, d'une problématisation de l'enjeu, d'une théorie du changement social, d'une conception des solutions (Halpern et Le Galès, 2011) et de la régulation gouvernants/gouvernés (Lascoumes et Simard, 2011). L'approche par les instruments propose ainsi d'aborder les modalités concrètes de l'exercice du pouvoir dans toute leur diversité.

Les IAP invitent à sortir du prisme des technologies efficaces (Lascoumes et Simard, 2011) pour comprendre les raisons qui influencent leur choix et la manière dont ils orchestrent les relations sociales et de pouvoir sur un territoire. Ils ont des effets propres, contraignent les acteurs et ouvrent un potentiel de reconfigurations des rapports de force.

Basée sur un travail empirique, l'approche par les IAP propose de décaler le regard pour aborder les formes de pilotage des sociétés complexes et penser l'action publique de manière «transsectorielle» (Halpern et Jacquot, 2015). Un des reproches adressés à cette approche est de sous-estimer notamment les contraintes des configurations et des structures institutionnelles existantes qui pèsent sur le choix des instruments (Halpern et al., 2014). Pour autant, ces derniers permettent de mettre en évidence les projets politiques souvent implicites qui les sous-tendent.

Appliquée à l'adaptation, l'approche par les IAP permet d'investiguer les arbitrages sociaux, économiques et politiques en jeu, les reconfigurations politiques en germe et l'attribution des responsabilités financières et institutionnelles dans les débats sur le choix des instruments. Alors que les IAP renvoient plutôt à des dispositifs économiques et gestionnaires, nous y intégrons aussi les infrastructures au sens propre (digues, par exemple). Comme c'est le cas pour d'autres instruments, les décisions sur les infrastructures intègrent, en effet, une certaine représentation des enjeux et ont des effets sur le territoire et le jeu d'acteurs.

Pour mieux caractériser les conceptions de la régulation politique, Lascoumes et Le Galès ont distingué, selon les instruments, des types de rapport au politique et des types de légitimité. Les instruments législatifs et réglementaires s'inscrivent dans un certain type de rapport politique où l'Etat se pose en tuteur du social; les instruments économiques et sociaux correspondent à un État redistributeur; les instruments conventionnels et incitatifs à un État mobilisateur ; les instruments informatifs à la démocratie du public et les normes et standards à des mécanismes de marché. De son côté, Hassenteufel (2008) a revisité la typologie des politiques de Lowi (1972) en associant des types de politique emblématique à des formes d'État (l'État régalien, nation, providence, producteur et régulateur) et les modes de légitimation (maintien de l'ordre, valeurs collectives, prise en charge des risques collectifs, bien-être économique, efficience et démocratie).

Nous nous référerons également aux répertoires d'action mis en exergue par Charles Tilly (1984). L'usage de ces répertoires d'action collective - qui, comme un répertoire de jazz, permettent des improvisations - se fait sous la contrainte de la situation et de la concurrence avec les autres groupes et dépend des ressources dont disposent les acteurs.

\section{L'adaptation à l'épreuve du terrain : une proposition de typologie}

Nous avons construit une typologie des adaptations locales aux changements globaux à partir d'une investigation empirique des logiques et des projets politiques qui les portent à l'échelle de plusieurs territoires de SCOT et bassins versants côtiers, lesquels sont envisagés comme des systèmes écologiques et sociaux (SES). Alors que l'analyse sous cet angle s'initie par la dimension 
écologique, l'approche par le territoire la fait démarrer par l'appréhension des dimensions sociales; ces deux approches ayant un intérêt commun pour les systèmes d'interactions entre des composantes de différentes natures, incluant le social et l'écologique (Barreteau et al., 2016).

\section{Envisager l'adaptation comme un processus}

Représenter l'adaptation comme un processus amène à s'intéresser à la trajectoire du SES, notamment à l'évolution de sa vulnérabilité (Magnan et al., 2012). Bien que, dans la réalité, les faits ne suivent pas forcément une suite logique et cohérente, le processus d'adaptation, qu'il prenne en compte un choc, une perturbation graduelle, des perturbations répétées ou des signaux annonciateurs de perturbation à venir, rassemble des éléments d'organisation temporelle communs : phase préadaptative, occurrence du changement ou des signaux, phase transitoire avec examen des réponses possibles et de leur efficacité attendue, mise en œuvre. Le processus d'adaptation reste continu et n'est pas nécessairement linéaire. Cette représentation permet de déconstruire la fabrique sociopolitique des solutions et des choix pour infléchir la trajectoire du SES.

\section{Construction de la typologie à partir d'une étude de cas sur la côte languedocienne}

Nous construisons notre typologie à partir d'un terrain littoral principal et l'avons consolidée avec des terrains secondaires. Notre terrain principal s'étend de la métropole montpelliéraine jusqu'à la petite Camargue, avec 4 syndicats mixtes de SCOT et 5 syndicats mixtes ou établissements publics territoriaux de bassin (EPTB). Il compte plusieurs bassins versants côtiers et une côte sableuse. Les multiples enjeux qui affectent le fonctionnement du SES dépassent les frontières administratives classiques et l'analyse de ce système permet d'observer les interactions entre ces enjeux. En effet, le territoire est fortement urbanisé, en particulier sur la zone côtière, avec des hotspots de biodiversité. Marquée par une activité touristique importante, fruit d'un fort interventionnisme de l'Etat dans les années 1960, son économie repose également sur l'agriculture (viticulture, marâ̂chage) et un secteur résidentiel lié à une politique d'accueil favorisant l'installation de populations nouvelles. Le territoire est soumis à de fortes inondations, une érosion graduelle du trait de côte, des submersions côtières et une pression sur la ressource en eau. Ces pressions s'intensifient avec le changement climatique et l'important afflux démographique. Les plans littoraux de prévention du risque inondation du Languedoc-Roussillon ont récemment intégré la perspective du changement climatique à la suite du Plan national d'adaptation au changement climatique (PNACC) de 2011 ${ }^{7}$. Le schéma directeur d'aménagement et de gestion des eaux (SDAGE) Rhône Méditerranée Corse 2016-2021 en a fait son orientation fondamentale. Ainsi, ces territoires côtiers sont particulièrement propices pour observer les adaptations locales du fait de leur exposition particulièrement saillante aux changements globaux et du croisement des aléas attendus (montée du niveau de la mer, érosion côtière, salinisation des nappes côtières, saturation des espaces). Nos terrains secondaires ont été investigués avec une posture d'observation participante lors de missions de conseil. Ils sont constitués par un syndicat mixte côtier, la ville de Paris et des intercommunalités rurales des Hautsde-France.

Nous avons réalisé 62 entretiens auprès d'élus, de techniciens de l'État et de collectivités locales, d'acteurs associatifs et économiques, à différentes échelles (communale à régionale $)^{8}, 8$ observations participantes de réunions de concertation, de séminaires techniques en lien avec l'adaptation entre 2014 et 2016. Nous avons également procédé à une première analyse des actions inscrites dans les stratégies et les plans d'action (schémas de cohérence territoriale, schémas d'aménagement et de gestion des eaux, programmes d'action de prévention des inondations, plans climat). Cette première analyse a consisté à repérer dans ces discours à quoi se référait explicitement le fait ou la capacité de s'adapter: adaptation «de qui» ou «de quoi», «à quoi», et à quelles échelles temporelles et spatiales. Nous avons ensuite confronté ces éléments aux adaptations évoquées et caractérisées comme telles par les acteurs interviewés. De manière inductive, nous avons à cet effet procédé à une analyse thématique et textuelle du corpus des changements, des instruments mentionnés par les interviewés et les documents, et des argumentaires utilisés.

\footnotetext{
${ }^{7}$ Le PNACC de 2011 recommande la prise en compte d'une hypothèse d'élévation du niveau de la mer à l'horizon 2100 lors de l'élaboration ou de la révision de plans de prévention des risques (PPR) littoraux. En Languedoc-Roussillon, l'élaboration des PPR submersion marine a fait l'objet d'une doctrine réalisée par la direction régionale de l'Environnement, de l'Aménagement et du Logement (Dreal). Celle-ci prend en compte l'aléa 2100 dans l'étude des aléas, les prescriptions et la cartographie.

${ }^{8}$ Les entretiens ont été conduits par S. Dhénain. Nous n'avons pu obtenir d'entretiens avec les opérateurs touristiques importants.
} 


\section{Proposition d'une typologie interdisciplinaire}

Notre typologie repose ainsi sur 3 dimensions principales :

- Une qualification plus précise des actions énonçant l'adaptation de quoi/qui, à quoi, les échelles spatiales et temporelles en jeu et le degré de transformation du SES qu'elles ambitionnent. À partir de la littérature présentée ci-dessus, nous distinguons l'accommodation, l'ajustement, la transformation, auxquels nous ajoutons la résistance aux changements subis;

- Le recensement des instruments d'action publique qui visent à opérationnaliser l'adaptation;

- L'identification des rhétoriques mobilisées et, à travers elles, l'analyse des logiques politiques (s'adapter pour quoi faire?) et des modes de régulation des rapports entre l'État et les acteurs qui apparaissent implicitement derrière les instruments.

Ainsi, quatre ensembles cohérents distinguent les adaptations selon une certaine logique : a) «Contrôler et maintenir» qui s'apparente à de la résistance aux perturbations ; b) «Faire faire» qui consiste à s'accommoder et à pousser les autres acteurs à l'action; c) «Réguler» qui relève de l'ajustement; d) «Reconfigurer» qui est caractérisé par une volonté plus manifeste des acteurs de transformer le système. Chacune de ces logiques politiques mobilise et combine certains types d'instruments avec des objectifs affichés ou sous-jacents distincts. Les types d'adaptation identifiés s'articulent autour des quatre grandes logiques suivantes.

\section{Contrôler et maintenir}

La logique «Contrôler et maintenir» se caractérise par une résistance aux changements. Elle s'appuie sur des instruments tels que des solutions d'ingénierie, des équipements et des infrastructures visant à protéger le territoire contre les risques naturels, à sécuriser les modes de vie actuels face aux évolutions tendancielles, mais surtout face aux extrêmes observés jusqu'ici. L'objet de l'action d'adaptation est de répondre à des risques spécifiques (inondations, érosion, mais aussi sécheresse) et de réduire les pertes matérielles et économiques. Une forte croyance en l'expertise scientifique et le progrès technique transparaît dans les discours. Comme le dit un représentant de la Dreal : «L'idée portée [par le président du conseil régional] était que nous sommes une région attractive [...] nous faisons le pari d'accueillir ces populations. [...] La région a dit: essayons de lever les facteurs limitants, dont l'eau ». On fait alors appel à la technique : digues, épis et brise-lames, ou grands projets d'aménagement tels que Aqua Domitia (projet d'infrastructure hydraulique de transfert d'eau depuis le Rhône).
Cela passe aussi par des instruments réglementaires, tels les plans de prévention des risques littoraux (PPRL), plus restrictifs sur les ouvertures à urbanisation. L'État est appelé ici, au nom de l'intérêt général, à jouer son rôle régalien d'application de la loi, à établir des règles de droit, à « dire » et «à prévenir» le risque (Gilbert, 2013) afin de maintenir les trajectoires actuelles. Les actions requièrent un portage à une échelle large: l'État, la région ou un syndicat mixte appelant l'État pour le financement sont maîtres d'ouvrage. Toutefois, ces actions ont des coûts financiers importants et exponentiels (entretien des ouvrages) dans un contexte où le rôle de l'État a largement évolué, notamment avec la loi GEMAPI $^{9}$ et la réduction des financements publics. Ces actions génèrent un déplacement spatial (amont-aval ou le long de la côte) et temporel (les digues ont un effet dans le temps, générant un transfert sédimentaire), des sédiments pouvant être sources de «maladaptations ${ }^{10}$ ». Bien que les acteurs porteurs de cette logique aient conscience des effets dommageables, c'est la nécessité de préserver à court terme les intérêts économiques conséquents en jeu qui prime.

\section{Faire faire}

Cette deuxième logique est celle du «porter à connaissance», avec des instruments tels que des observatoires, des livres blancs, des études de vulnérabilités (dans le cadre des démarches Plan climat ou du contrat de plan État-région [CPER] 2007-2013 de l'exrégion Languedoc-Roussillon, par exemple) ou des systèmes d'alerte précoce. Le ressort de l'action est cognitif et vise à donner à voir l'état du territoire pour adapter les représentations des décideurs, des citoyens et des acteurs locaux et les amener à (ré)agir et à évoluer. Autrement dit, il faut rendre tangible l'intangible: la vulnérabilité du territoire, actuelle et future, ainsi que les avenirs climatiques probables. La connaissance cherche à circonscrire les «bonnes" informations à prendre en compte - scénarios tendanciels et/ou seuils de crise-, mais sert également à mettre le problème à l'agenda des autres acteurs aux échelles subsidiaires et à énoncer des standards de bonnes pratiques ${ }^{11}$. L'initiative de l'action est renvoyée aux autres acteurs. Dans un contexte où les

\footnotetext{
${ }^{9}$ Loi n ${ }^{\circ} 2017-1838$ du 30 décembre 2017 relative à l'exercice des compétences des collectivités territoriales dans le domaine de la gestion des milieux aquatiques et de la prévention des inondations, Journal Officiel de la République française, 0305, 31 décembre 2017.

${ }^{10}$ Les «maladaptations » sont définies comme des actions qui accentuent la vulnérabilité d'autres secteurs, systèmes et groupes sociaux (Barnett et O'neill, 2010).

${ }^{11}$ Par exemple, le site littoral.languedocroussillon.fr décrit non seulement les vulnérabilités du littoral étudiées mais énonce les bonnes pratiques en matière de recul stratégique.
} 
finances publiques sont sous tension, on observe le renvoi de la maîtrise d'ouvrage vers les communes ou les établissements publics de coopération intercommunale (EPCI), voire vers les individus. Par exemple, les abonnements souscrits à des systèmes d'alerte visent à améliorer la capacité des individus à accepter les crises et à y faire face (Quenault, 2015).

L'État s'inscrit ici dans la posture de celui qui pilote à distance et établit des standards de bonnes pratiques (Epstein, 2012), comme l'indique un représentant du secrétariat général pour les affaires régionales (SGAR): «Côté État, il y a un regard expert, mais sur ce qui est projeté, c'est les collectivités qui décident et [qui] portent». L'expert tient une place importante dans les instruments qui répondent à cette logique, des instruments qui sont portés par tous les types d'acteurs institutionnels, ce qui peut conduire à une dilution de la responsabilité.

\section{Réguler}

La troisième logique repérée cherche à accepter et à réguler les processus de changement, en amortir les effets, favoriser la résilience du territoire et repousser les seuils au-delà desquels le système basculerait. L'adaptation passe par celle des modes de vie et de consommation (comme les consommations d'eau) et des modes de gestion (passage à une gestion dite « douce» de l'érosion littorale). Les acteurs reconnaissent la finitude des ressources et la responsabilité humaine dans les pressions exercées sur celles-ci ( On se retrouve ici avec un taux de fragilisation qu'on a provoqué», déclare un représentant du SGAR). Il s'agit de rendre compatibles développement économique et préservation des ressources, de trouver un nouvel équilibre dans la gestion de la ressource ( «fonder une nouvelle harmonie entre ville et nature», dixit un acteur du SCOT de Montpellier), et donc de nouveaux compromis («promouvoir un nouvel aménagement "d'équilibres" », dit un acteur du SCOT du Pays de l'Or). Cela passe par des instruments procéduraux (l'ajustement des modes de répartition de la ressource en eau et la priorisation des usages en cas de sécheresse), incitatifs (les kits d'économie d'eau), réglementaires (l'introduction d'un nouveau zonage pour les plans de prévention du risque inondation [PPRI] littoraux en Languedoc-Roussillon ${ }^{12}$ ), ou encore des instruments techniques plus respectueux des milieux écologiques (les modes doux de gestion du littoral). Comme le souligne un technicien de la région: «On va restaurer un fonctionnement naturel de la plage». On

\footnotetext{
${ }^{12}$ Pour intégrer l'horizon 2100 dans la cartographie des risques, des «zones de précaution urbaine» ont été introduites dans les PPRI de notre terrain. Elles sont concernées par l'aléa 2100, qui intègre le scénario moyen d'élévation du niveau de la mer (les constructions sont autorisées mais sous contraintes).
}

trouve également des opérations urbaines (par exemple de désimperméabilisation).

Les porteurs de cette catégorie sont l'État, au niveau régional, et majoritairement des collectivités locales infrarégionales. Pour ces dernières, les compétences et les capacités financières et institutionnelles ne sont pas toujours à l'échelle des enjeux. On observe un renouvellement des instruments vers ceux de la modernisation écologique et une évolution du registre de légitimation de l'action (capacités de charge d'un territoire, volumes prélevables pour l'eau).

\section{«Reconfigurer»}

Ce dernier type ouvre la possibilité, voire la nécessité, de transformations des équilibres spatiaux, sociaux, économiques et écologiques plus marquées et des bifurcations plus franches des trajectoires. Ce sont les territoires, leurs modes de vie et de développement et leurs configurations spatiales que l'on cherche à transformer au regard des évolutions tendancielles, mais aussi des «surprises» climatiques possibles. Des pertes sont effectivement envisagées, voire acceptées (pertes d'espaces, par exemple). Dans le cas de la relocalisation, le déplacement d'enjeux (routes, activités économiques, habitations) et le réagencement $\mathrm{du}$ territoire sont discutés. L'ambition est de «changer de modèle», de sortir de la «mono-industrie du tourisme», en trouvant une complémentarité entre littoral et arrière-littoral et en diversifiant les activités économiques (œnotourisme, tourisme vert, etc.). Plusieurs instruments sont combinés dans une même opération. Le recul stratégique propose d'associer instruments communicationnels, juridiques, économiques et de gouvernance. L'échelle spatiale de l'action dépasse les zones côtières et embrasse le rétrolittoral. Le portage potentiel est multiacteurs. Cela induit une grande complexité, liée notamment à la nécessaire coordination entre acteurs. Comme nous le raconte un technicien de collectivité locale : «Il y a un élément qui se fait bon an, mal an, c'est du dialogue [...] c'est très lent : c'est le recul stratégique. L'idée c'est de le faire en gardant un potentiel économique malgré ça [la montée du niveau de la mer], c'est repenser l'occupation du territoire sur toute la plaine littorale ». Sur notre terrain, le recul d'enjeux (en matière de routes) est opéré mais le recul stratégique est, pour le moment, seulement mis en débat. Toutefois, cette catégorie ne renvoie pas uniquement au recul stratégique face à l'érosion côtière, bien qu'il soit le plus cité ${ }^{13}$.

\footnotetext{
${ }^{13}$ Par exemple, la ville de Nîmes met en place une opération qui adapte le projet urbain au risque inondation, en faisant le choix de ne pas se protéger contre l'événement de référence. La transformation réside dans le choix d'un changement de trajectoire pour le territoire pour vivre avec le risque.
} 
Tab. 1. Typologie détaillée des actions d'adaptation (source: S. Dhénain, O. Barreteau).

\begin{tabular}{|c|c|c|c|c|}
\hline & Résistance & Accomodation & Ajustement & Transformation \\
\hline Type & Contrôler et maintenir & Faire faire & Réguler & Reconfigurer \\
\hline Mots-clés & $\begin{array}{l}\text { Sécuriser le territoire } \\
\text { (et les modes } \\
\text { de développement actuels) }\end{array}$ & Faire faire le territoire & Ménager le territoire & $\begin{array}{l}\text { Déménager/réagencer le } \\
\text { territoire }\end{array}$ \\
\hline Instruments & $\begin{array}{l}\text { Aquadomitia } \\
\text { Digues } \\
\text { Cartographie des PPRL } \\
\text { avec durcissement } \\
\text { des règles d'urbanisation }\end{array}$ & $\begin{array}{l}\text { Observatoires, } \\
\text { Études de vulnérabilités } \\
\text { Livres blancs }\end{array}$ & $\begin{array}{l}\text { Modes de gestion doux, } \\
\text { Renouvellement des modes } \\
\text { de répartition de la ressource } \\
\text { en eau, Projets urbains }\end{array}$ & Recul stratégique \\
\hline $\begin{array}{l}\text { Adaptation } \\
\text { de quoi }\end{array}$ & Infrastructures techniques & $\begin{array}{l}\text { Connaissances } \\
\text { Représentations }\end{array}$ & $\begin{array}{l}\text { Modes de vie } \\
\text { Modes de consommation, } \\
\text { Modes de gestion }\end{array}$ & $\begin{array}{l}\text { Territoires et leurs } \\
\text { modes de fonctionnement, } \\
\text { Modèles économiques, } \\
\text { Modes de gestion } \\
\text { et gouvernance }\end{array}$ \\
\hline $\begin{array}{l}\text { Logique } \\
\text { politique }\end{array}$ & $\begin{array}{l}\text { Résister, protéger } \\
\text { Logiques interventionnistes }\end{array}$ & $\begin{array}{l}\text { Inciter les autres } \\
\text { acteurs à agir } \\
\text { Logiques de pilotage } \\
\text { à distance }\end{array}$ & $\begin{array}{l}\text { Ajuster } \\
\text { Localisme }\end{array}$ & $\begin{array}{l}\text { Transformer } \\
\text { Logiques redistributives? }\end{array}$ \\
\hline
\end{tabular}

Plusieurs pas de temps sont pris en compte pour l'action (court, moyen et long terme). Les choix à opérer conduisent à des tensions et à des conflits potentiels (où reculer? Quels enjeux protéger?) et les coûts économiques et politiques sont importants. La question «qui paie quoi et pour quoi faire» pose de manière plus saillante encore celle des compromis, des arbitrages et des collaborations. Comme pour les autres catégories décrites précédemment, elle pousse de fait à la remise en cause du sens politique du projet territorial.

\section{Une pluralité de logiques politiques sous-jacentes \\ Un répertoire d'action pluriel mais sous contraintes}

Notre étude montre qu'il existe une pluralité de registres de réponses face aux changements globaux qui sont souvent combinés. À travers les quatre catégories mises en avant dans la typologie (Tab. 1), il apparaît que les instruments mobilisés et les leviers activés diffèrent. La logique «Faire faire» mobilise des instruments cognitifs et informationnels visant à faire évoluer les représentations du territoire, des risques, des vulnérabilités et à faire agir les autres. Dans la logique «Contrôler et maintenir », des instruments techniques et réglementaires sont utilisés pour protéger les biens et les activités.
Des instruments conventionnels, incitatifs, réglementaires et techniques dans la logique «Réguler» visent à ajuster les modes de vie et de développement pour amortir les pressions. Enfin, des instruments multiples (gouvernance, juridiques, économiques, cognitifs) sont combinés dans une même opération dans la dernière logique, «Reconfigurer ». Cette complexité vient s'ajouter aux coûts financiers, économiques et aux conséquences sociales de ces opérations.

Si la première logique maintient le projet territorial dans un statu quo, la quatrième nécessite de le repenser. Cette dernière, porteuse de transformations radicales du système socioécologique, ne se rencontre guère sur notre terrain. La complexité de sa mise en œuvre combine une action associant des acteurs, publics et privés, à des échelles spatiales multiples et aux logiques d'action et intérêts différents, voire divergents. Elle nécessite un travail plus important de négociation entre ces acteurs du fait de choix de ruptures avec les registres d'action en cours.

Ces répertoires se distinguent ainsi par l'échelle spatiale à laquelle les actions sont mises en œuvre. Le registre «Reculer et reconfigurer » nécessite une collaboration multiniveaux entre de nombreuses institutions intervenant à différentes échelles. Le registre «Contrôler et maintenir », du fait de l'envergure des actions, requiert un portage de l'action à grande échelle (intercommunale, régionale ou nationale). Les dimensions temporelles 
diffèrent aussi. Le registre «Contrôler et maintenir » prend plutôt en compte les extrêmes passés; le registre «Réguler» s'intéresse aux évolutions à venir, mais dans le court terme; le registre «Reculer et reconfigurer» appréhende différents pas de temps : court, moyen et long terme.

Sur le terrain, les répertoires d'action publique ne sont pas exclusifs les uns des autres et sont combinés sur un même territoire. Un même territoire peut passer d'un type à un autre sans ordre a priori et sans que cela soit irréversible. Ce répertoire que constituent les différents types combinables entre eux permet d'improviser face aux changements perçus ou attendus (Berdoulay et Soubeyran, 2012). Son usage est, toutefois, contraint (Tilly, 1984). La logique "Contrôler et maintenir» est observée sur des territoires à forts enjeux économiques (par exemple, en lien avec «la mono-industrie» du tourisme). Les adaptations de type «Réguler» se retrouvent davantage sur des territoires très contraints par des pressions sur les ressources (par exemple, dans le cas de milieux urbanisés avec la présence de nombreux réseaux, infrastructures, etc.). Certains territoires ont des marges de manœuvre plus importantes dans leur choix d'instruments relevant du type «Réguler». C'est le cas de ceux qui ont accès à des ressources financières, avec des élus porteurs, entrepreneurs de politiques publiques. La logique «Faire faire» se retrouve sur tout type de territoire. Le quatrième type «Reconfigurer» est peu mis en œuvre sur notre terrain, mais il est présent comme objet de débat. Les discussions sur le sujet sont plus approfondies sur des terrains proches du littoral (Valras, Vias), lorsque le territoire doit faire face à une perte (d'habitations, d'espaces récréatifs, d'activités économiques) et que des espaces sont menacés dans le court terme. Néanmoins, l'événement catastrophique n'est pas l'unique déclencheur du passage vers la transformation, d'autres éléments sont nécessaires, comme la préparation de la reconfiguration du territoire (Moatty, 2015).

\section{Derrière les types d'adaptation, des configurations politiques différentes}

Notre typologie montre que les instruments ne sont pas neutres. Ils sont porteurs d'une logique de régulation politique, d'attributions différentes de responsabilités des actions et de leur financement. La logique « Contrôler et maintenir » appelle à un État régalien et à une prise en charge collective des risques. Mais le rôle de l'État ayant évolué et ses financements diminué, celui-ci renvoie le portage de l'action vers les acteurs locaux adossés à des cofinancements (européens, par exemple). «Faire faire » s'inscrit dans une logique étatique de pilotage à distance par le biais des standards (Epstein, 2005) et de la démocratie du public (Lascoumes et Le Galès, 2005). La responsabilité de l'action et le coût financier sont reportés sur les collectivités et les acteurs locaux, voire les individus (via l'assurance individuelle). Dans le type «Réguler», le niveau local prend en charge la maitrise d'ouvrage de l'action. L'État ne pilote plus la démarche mais il est régulateur et animateur, proposant, par exemple, aux territoires des appels à projet. L'action est construite sur la base d'une solidarité horizontale, mais aussi de partenariats public-privé ou du recours à des cofinancements nationaux ou européens. Dans le cadre d'une mise en concurrence des territoires, la logique « Réguler» est mise en œuvre par des territoires cherchant à se différencier à travers des projets innovants (Pinson, 2005; Pinson, 2009). Par exemple, certaines villes expérimentent la végétalisation pour réduire le ruissellement et l'îlot de chaleur urbain, ou encore la désimperméabilisation de certaines zones. La métropole montpelliéraine met en avant son projet urbain «Ode à la mer» comme une programmation pionnière d'actions répondant aux enjeux de l'adaptation. Le dernier type «Reconfigurer» nécessiterait un partage des responsabilités, une maîtrise d'ouvrage à une échelle large et multiniveaux.

\section{Conclusion}

Les adaptations, ces actions entreprises par les acteurs pour formuler une réponse aux changements globaux en cours ou anticipés, sont des processus continus qui mettent en jeu des visions du territoire et de sa trajectoire souhaitable différentes, divergentes, voire conflictuelles. L'adaptation génère des modifications du système plus ou moins profondes et le terme générique d'adaptation recouvre un éventail de reconfigurations.

L'adaptation est plurielle, elle renvoie en fait à un répertoire d'actions publiques diversifié. Les acteurs d'un système socioécologique cherchent à opérationnaliser des réponses à travers le choix d'instruments d'action publique et de leurs modalités de mise en œuvre. Ceux-ci ne sont pas l'application mécanique de principes ou de recettes toutes faites, mais le fruit de représentations, de logiques et d'arbitrages des acteurs dans un contexte social, économique, écologique et politique spécifique. C'est ce que nous avons montré en proposant une typologie qui permet de rendre visibles les choix sous-jacents aux politiques d'adaptation, la combinaison de ces logiques dépendant du jeu des acteurs et des contraintes du territoire. Autrement dit, loin d'être une problématique purement technique, les adaptations sont politiques ; une dimension qui, hélas, est souvent laissée dans l'ombre. Alors que le bien-fondé de l'adaptation est souvent présenté comme allant de soi, il y a nécessité de mettre en débat la diversité des visions qui la soustendent, notamment pour éviter ensuite son rejet massif. 


\section{Remerciements}

Nous remercions l'Agence de l'eau Rhône-MéditerranéeCorse, l'Association nationale de la recherche et de la technologie (ANRT) et l'Agence nationale de la recherche (ANR) [projet MAGIC] pour leur soutien à ce travail.

\section{Références}

Adger W.N., 2006. Fairness in adaptation to climate change, Cambridge (MA), The MIT Press.

Aykut S.C., Dahan A., 2014. La gouvernance du changement climatique. Anatomie d'un schisme de réalité, in Pestre D. (Ed.), Le gouvernement des technosciences. Gouverner le progrès et ses dégâts depuis 1945, Paris, La Découverte, 97-132.

Barnett J., O'neill S., 2010. Maladaptation, Global Environmental Change, 20, 2, 211-213, doi: 10.1016/j.gloenv cha.2009.11.004.

Barreteau O., Giband D., Schoon M., Cerceau J., Declerck F., Ghiotti S., James T., Masterson V., Mathevet R., Rode S., 2016. Bringing together social-ecological system and territoire concepts to explore nature-society dynamics, Ecology and Society, 21, 4, 42, doi: 10.5751/ES-08834-210442.

Bassett T.J., Fogelman C., 2013. Déjà vu or something new? The adaptation concept in the climate change literature, Geoforum, 48, 42-53, doi: 10.1016/j.geoforum.2013.04.010.

Berdoulay V., Soubeyran O., 2012. Pratiques réflexives en aménagement pour une adaptation aux changements environnementaux, L'Espace géographique, 41, 2, 169180, doi: $10.3917 /$ eg.412.0169.

Berkes F., Folke C., 1998. Linking social and ecological systems for resilience and sustainability, in Berkes F., Folke C. (Eds), Linking social and ecological systems. Management practices and social mechanisms for building resilience, Cambridge, Cambridge University Press, 13-20.

Berkes F., Colding J., Folke C., 2008. Navigating socialecological systems. Building resilience for complexity and change, Cambridge, Cambridge University Press.

Biagini B., Bierbaum R., Stults M., Dobardzic S., McNeeley S. M., 2014. A typology of adaptation actions: a global look at climate adaptation actions financed through the Global Environment Facility, Global Environmental Change, 25, 97-108, doi: 10.1016/j.gloenvcha.2014.01.003.

Biesbroek G.R., Swart R.J., Carter T.R., Cowan C., Henrichs T., Mela H., Morecroft M.D., Rey D., 2010. Europe adapts to climate change: comparing national adaptation strategies, Global Environmental Change, 20, 3, 440-450, doi: 10.1016/j.gloenvcha.2010.03.005.

Bijlsma L., Ehler C., Klein R., Kulshrestha S., Mclean R., Mimura N., Nicholls R., Nurse L., Nieto H.P., Stakhiv E., Turner R.K., Warrick R.A., 1995. Coastal zones and small islands, in IPCC, Climate change, 1995. Impacts, adaptations, and mitigation of climate change: scientifictechnical analyses, [Contribution of Working Group II to the Second Assessment. Report of the Intergovernmental Panel on Climate Change, Watson R.T., Zinyowera M.C.,
Moss R.H. (Eds)], Cambridge, Cambridge University Press, 289-324.

Brooks N., 2003. Vulnerability, risk and adaptation: a conceptual framework, Tyndall Centre for Climate Change Research Working Paper, 38.

Brooks N., Adger W.N., Kell P.M., 2005. The determinants of vulnerability and adaptive capacity at the national level and the implications for adaptation, Global Environmental Change, 15, 2, 151-163, doi: 10.1016/j.gloenvcha.2004.12.006.

Burton I., Huq S., Lim B., Pilifosova O., Schipper E.L., 2002. From impacts assessment to adaptation priorities: the shaping of adaptation policy, Climate Policy, 2, 2, 145-159, doi: 10.3763/cpol.2002.0217.

Burton I., Kates R.W., White G.F., 1993. The environment as hazard, New York, Guilford Press.

Carpenter S., Walker B., Anderies J.M., Abel N., 2001. From metaphor to measurement: resilience of what to what?, Ecosystems, 4, 8, 765-781, doi: 10.1007/s10021-001-0045-9.

Cutter S.L., Mitchell J.T., Scott M.S., 2000. Revealing the vulnerability of people and places: a case study of Georgetown County, South Carolina, Annals of the Association of American Geographers, 90, 4, 713-737, doi: 10.1111/0004-5608.00219.

Demeritt D., 2001. The construction of global warming and the politics of science, Annals of the Association of American Geographers, 91, 2, 307-337, doi: 10.1111/00045608.00245.

Epstein R., 2005. Gouverner à distance. Quand l'État se retire des territoires, Esprit, 11, 96-111.

Epstein R., 2012. De la différenciation territoriale à la libre conformation, in Douillet A.-C., Faure A., Halpern C. (Eds), L'action publique locale dans tous ses états. Différenciation et standardisation, Paris, L'Harmattan, 127-138.

Folke C., 2006. Resilience: the emergence of a perspective for social-ecological systems analyses, Global Environmental Change, 16, 3, 253-267, doi: 10.1016/j.gloenvcha.2006.04.002.

Folke C., Hahn T., Olsson P., Norberg J., 2005. Adaptive governance of social-ecological systems, Annual Review of Environment and Resources, 30, 441-473, doi: 10.1146/ annurev.energy.30.050504.144511.

Gallopín G.C., 2006. Linkages between vulnerability, resilience, and adaptive capacity, Global Environmental Change, 16, 3, 293-303, doi: 10.1016/j.gloenvcha.2006.02.004.

Gilbert C., 2013. De l'affrontement des risques à la résilience. Une approche politique de la prévention, Communication \& langages, 176, 65-78.

Gunderson L.H., 2000. Ecological resilience - in theory and application, Annual Review of Ecology, Evolution, and Systematics, 31, 425-439, doi: 10.1146/annurev.ecol sys.31.1.425.

Gunderson L.H., Holling C.S., Peterson G.D., 2002. Surprises and sustainability: cycles of renewal in the Everglades, in Gunderson L.H., Holling C.S. (Eds), Panarchy: understanding transformations in human and natural systems, London, Island Press, 315-332.

Halpern C., Jacquot S., 2015. Aux frontières de l'action publique: l'instrumentation comme logique de (dé)sectorisation, in Boussaguet L., Jacquot S., Ravinet P. (Eds), Une 
"French touch" dans l'analyse des politiques publiques?, Paris, Presses de Sciences Po, 57-84.

Halpern C., Lascoumes P., Le Galès P. (Eds), 2014. L'instrumentation de l'action publique: controverses, résistances, effets, Paris, Presses de Sciences Po.

Halpern C., Le Galès P., 2011. Pas d'action publique autonome sans instruments propres. Analyse comparée et longitudinale des politiques environnementales et urbaines de l'Union européenne, Revue française de science politique, 61, 1, 51-78, doi: 10.3917/rfsp.611.0051.

Hassenteufel P., 2008. Sociologie politique : l'action publique, Paris, Armand Colin.

Huq S., Reid H., Konate M., Rahman A., Sokona Y., Crick F., 2004. Mainstreaming adaptation to climate change in least developed countries (LDCs), Climate Policy, 4, 1, 25-43.

Kasperson R.E., Archer E.R., 2005. Vulnerable peoples and places, in Hassan R.M., Scholes R., Ash N. (Eds), Ecosystems and human well-being. Current state and trends: findings of the Condition and Trends Working Group of the Millenium Ecosystem Assessment, London, Island Press, 143.

Kelly P.M., Adger W.N., 2000. Theory and practice in assessing vulnerability to climate change and facilitating adaptation, Climatic Change, 47, 4, 325-352, doi: 10.1023/ A:1005627828199.

Keskitalo E.C.H., Kulyasova A.A., 2009. The role of governance in community adaptation to climate change, Polar Research, 28, 1, 60-70, doi: 10.1111/j.1751-8369.2009.00097.x.

Lascoumes P., Le Galès P. (Eds), 2005. Gouverner par les instruments, Paris, Presses de Sciences Po.

Lascoumes P., Simard L., 2011. L'action publique au prisme de ses instruments, Revue française de science politique, 61, 1 , 5-22, doi: 10.3917/rfsp.611.0005.

Lowi T.J., 1972. Four systems of policy, politics, and choice, Public Administration Review, 32, 4, 298-310, doi: 10.2307/ 974990.

Magnan A., Duvat V., Garnier E., 2012. Reconstituer les «trajectoires de vulnérabilité » pour penser différemment l'adaptation au changement climatique, Natures Sciences Sociétés, 20, 1, 82-91, doi: 10.1051/nss/2012008.

Marquet V., Salles D., 2014. L'adaptation au changement climatique en France et au Québec. Constructions institutionnelles convergentes et diffusions contrastées, Critique internationale, 62, 1, 73-91, doi: 10.3917/crii.062.0073.

Moatty A., 2015. Pour une Géographie des reconstructions post-catastrophe: risques, sociétés et territoires. Thèse de doctorat en géographie et aménagement de l'espace, Montpellier, Université Paul-Valéry Montpellier.

Pielke R.A., 1998. Rethinking the role of adaptation in climate policy, Global Environmental Change, 8, 2, 159-170, doi: 10.1016/S0959-3780(98)00011-9.

Pinson G., 2005. Le projet urbain comme instrument d'action publique, in Lascoumes P., Le Galès (Eds), Gouverner par les instruments, Paris, Presses de Sciences Po, 199-233.
Pinson G., 2009. Gouverner la ville par projet. Urbanisme et gouvernance des villes européennes, Paris, Presses de Sciences Po.

Quenault B., 2015. De Hyōgo à Sendai, la résilience comme impératif d'adaptation aux risques de catastrophe : nouvelle valeur universelle ou gouvernement par la catastrophe?, Développement durable \& territoires, 6, 3, doi: 10.4000/ developpementdurable.11010.

Richard E., 2013. L'action publique territoriale à l'épreuve de l'adaptation aux changements climatiques: un nouveau référentiel pour penser l'aménagement du territoire? Thèse de doctorat en aménagement, Tours, Université de Tours.

Simonet G., 2016. De l'ajustement à la transformation : vers un essor de l'adaptation?, Développement durable \& territoires, 7, 2, doi: 10.4000/developpementdurable.11320.

Smit B., Burton I., Klein R.J.T., Street R., 1999. The science of adaptation: a framework for assessment, Mitigation and Adaptation Strategies for Global Change, 4, 3-4, 199-213, doi: 10.1023/A:1009652531101.

Smit B., Burton I., Klein R.J.T., Wandel J., 2000. An anatomy of adaptation to climate change and variability, Climatic Change, 45, 1, 223-251, doi: 10.1023/A:1005661622966.

Smit B., Pilifosova O., Burton I., Challenger B., Huq S., Klein R.J.T., Yohe G., Adger N., Downing T., Harvey E., Kane S., Parry M., Skinner M., Smith J., 2001. Adaptation to climate change in the context of sustainable development and equity, in IPCC, Climate change 2001. Impacts, adaptation, and vulnerability [Contribution of Working Group II to the Third Assessment Report of the Intergovernmental Panel on Climate Change, McCarthy J.J., Canziani O.F., Leary N.A., Dokken D.J., White K.S. (Eds)], Cambridge, Cambridge University Press, 877-912.

Smit B., Wandel J., 2006. Adaptation, adaptive capacity and vulnerability, Global Environmental Change, 16, 3, 282292, doi: 10.1016/j.gloenvcha.2006.03.008.

Tilly C., 1984. Les origines du répertoire d'action collective contemporaine en France et en Grande-Bretagne, Vingtième siècle, revue d'histoire, 4, 89-108, www.persee.fr/doc/ xxs_0294-1759_1984_num_4_1_1719.

Turner B.L., Kasperson R.E., Matson P.A., Mccarthy J.J., Corell R.W., Christensen L., Eckley N., Kasperson J.X., Luers A., Martello M.L., Polsky C., Pulsipher A., Schiller A., 2003. A framework for vulnerability analysis in sustainability science, Proceedings of the National Academy of Sciences of the United States of America, 100, 14, 8074-8079, doi: /10.1073/pnas.1231335100.

Walker B., Holling C.S., Carpenter S.R., Kinzig A., 2004. Resilience, adaptability and transformability in socialecological systems, Ecology and Society, 9, 2, 5, www. ecologyandsociety.org/vol9/iss2/art5/.

Wisner B., Blaikie P., Cannon T., Davis I., 2004. At Risk. Natural hazards, people's vulnerability and disasters, London/New York, Routledge.

Citation de l'article : Dhénain S., Barreteau O., 2018. Pluralité des instruments et des logiques politiques pour l'adaptation aux changements globaux en zone côtière. Nat. Sci. Soc. 26, 4, 407-417. 